\title{
Postoperative Hepatobiliary Surgeries' Fungal Infections Epidemiology: Prevalence and Risk Factors
}

\author{
WESAM S. MORAD, M.D. ${ }^{1}$; ESSAM EL-SHIMII, M.D. ${ }^{2}$; AMAL EL-SHARNOBY, M.D. ${ }^{\mathbf{3}}$ and \\ AMR MOSTAFA AZIZ, M.D. 4
}

The Departments of Epidemiology \& Preventive Medicine ${ }^{1}$, Hepatology ${ }^{2}$, Clinical Pathology and Hepatopancreato-Biliary Surgery ${ }^{4}$, National Liver Institute, Menoufia University

\begin{abstract}
Background: Candidemia and disseminated candidiasis are major causes of morbidity and mortality in hospitalized patients especially in ICU, the incidence of invasive candidiasis is on a steady rise because of increasing use of multiple antibiotics and invasive procedures carried out in the ICUs. Risk factors for invasive candidiasis \& candidaemia include prior antimicrobial therapy, central venous catheters, urinary catheters, ICU admission, parenteral nutrition, major surgery and immunosuppressive therapies. Candida species were the most frequently isolated organism from any sites and comprise $85 \%$ of total number of cultures.
\end{abstract}

Aim of Study: Assessing the rate of fungal infections after hepatobiliary surgery and identification of the risk factors associated with the development of fungal infections in such patients.

Patients and Methods: A prospective cohort hospital based study was carried out at National Liver Institute. The studied group consisted of 210 patients. All of them were examined thoroughly, their data were registered and sampled at two times, one at day of admission to be sure that they are free of fungal infection and the second was after hepatobiliary surgery pre-designed questionnaire was used which include data about personal history, medical history and suggested risk factors for fungal infections.

Results: In the present study the incidence rate of fungal infection among patients undergone hepatobiliary surgeries in NLI is $(45.2 \%)$ and the main predictors of fungal infection were age ( $p$-value 0.001$)$, antibiotic use ( $p$-value 0.05$)$, liver disease ( $p$-value 0.006$),$ CVC ( $p$-value 0.043$)$, urinary catheter $(p$-value 0.05$)$, and ICU hospitalization more than 48 hours ( $p$-value 0.000008$)$.

Conclusions: High incidence of fungal infection after hepatobiliary surgeries may reach $45 \%$ due to type of patient doing such surgeries. The great prevalence of fungal colonization inside ICU which is easily transmissible emphasizes very strongly on the importance of infection- control guidelines.

Recommendations: Aggressive antibiotics shouldn't be used outside ICU and only antibiotics according to culture

Correspondence to: Dr. Wesam Saber Morad, E-Mail: wesammorad@yahoo.com and sensitivity should be used to lessen down emergence of resistant strains and fungal flourishment.

Key Words: Intensive Care Unit-Central Venous Catheter - National Liver Institute.

\section{Introduction}

FUNGAL diseases pose an important threat to public health. Opportunistic infections such as cryptococcosis and aspergillosis are becoming increasingly problematic as the number of people with weakened immune systems rises. This group includes cancer patients, transplant recipients, other people taking medications that weaken the immune system, and people with HIV/AIDS. Hospitalassociated infections such as candidemia are the leading cause of bloodstream infections in the United States. Advancements and changes in healthcare practices can provide opportunities for new and drug-resistant fungi to emerge in hospital settings [1].

Community-acquired infections such as coccidioidomycosis (Valley fever), blastomycosis, and histoplasmosis, are caused by fungi that live in the environment in specific geographic areas. These fungi are sensitive to changes in temperature and moisture [1].

Candidemia and disseminated candidiasis are major causes of morbidity and mortality in hospitalized patients especially in the Intensive Care Units (ICU), the incidence of invasive candidiasis is on a steady rise because of increasing use of multiple antibiotics and invasive procedures carried out in the ICUs. Worldwide there is a shifting trend from C.albicans towards non albicans species, with an associated increase in mortality and antifungal resistance [2] 
Candida species were the most frequently isolated organism from any sites and comprise $85 \%$ of total number of cultures [3]

Risk factors for invasive candidiasis and candidaemia include prior antimicrobial therapy, central venous catheters, urinary catheters, ICU admission, parenteral nutrition, major surgery and immunosuppressive therapies. Candidemia in ICUs is associated with a considerable increase in hospital costs and length of hospital stay, so once fungal infection is confirmed, species identification is in most cases an effective method for prediction of antifungal susceptibility [4] .

\section{Patients and Methods}

A prospective cohort hospital based study which was conducted at National Liver Institute (NLI) during the period from the first of May 2014 to the end of December 2015. Number of patients included in this study was 210 undergo hepatobiliary surgeries of both sex and any age during the period from the first of August 2014 to August of 2015. All of them were examined thoroughly, their data were registered and sampled at two times, one at day of admission to be sure that they are free of fungal infection and the second was after hepatobiliary surgery, then sample identification; conventional methods for yeast and yeast-like isolates (Corn meal agar description, Germ tube formation, Urease test, finally cultured on HiCromeTM Candida Differential Media) and Morphological methods for filamentous isolates identification (slide culture technique and cultured on Candida Differential Media). Data were collected and statistically analyzed using SPSS (Statistical Package of Social Science) Version 20 and Epi Info 2000 programs applying both student's $t$-test and Mann-Whitney for quantitative variables, Chi squared test for qualitative variables with a significance level of $p \leq 0.05$. Logistic regression model was used to give adjusted odds ratio at $95 \%$ confidence interval of the effect of the different risk factors for the subject under the study.

\section{Results}

The study included 210 patients admitted to Surgery Department from August 2014 to August 2015 , their mean ages was $39.2 \pm 22.7$ years ranging from $0.15-73 y$. Most of the studied cases $(79 \%)$ were above 18 years of age and also it was noted that $56.7 \%$ of the total number of patients studied was male and $43.3 \%$ was female, $83.1 \%$ of patients above age of 18 were educated, $53.6 \%$ of patients above age of 18 were not working, $92.3 \%$ of patients above age of 18 were married and $62.9 \%$ of the all studied patients were from urban areas. Incidence rate of fungal infection among patients undergone major hepatobiliary surgeries in national liver institute is $(45.2 \%)$. Nearly $98 \%$ of positive cultures were of candida species only $2.1 \%$ noncandida (aspergillus) as $47.4 \%$ had c.glabrata infection, $41.1 \%$ had candida albican infection, $4.2 \%$ c.tropicalis, $3.1 \%$ mixed tropicalis and c.non albican, $2.1 \%$ mixed c.albican and c.non albican and $2.1 \%$ A.nigra.

Table (1): Socio-demographic risk factor in relation to fungal infection among patients undergoing hepatobiliary surgeries.

\begin{tabular}{|c|c|c|c|c|c|c|}
\hline \multirow{3}{*}{$\begin{array}{l}\text { Socio-demographic } \\
\text { risk factor }\end{array}$} & \multicolumn{4}{|c|}{ Fungal infection } & \multirow{3}{*}{$\begin{array}{c}\text { Chi } \\
\text { square } \\
\text { test } \\
p \text {-value }\end{array}$} & \multirow{3}{*}{$\begin{array}{c}\text { OR } \\
(95 \% \mathrm{CI})\end{array}$} \\
\hline & \multicolumn{2}{|c|}{$\begin{array}{l}\text { Positive } \\
(\mathrm{N}=95)\end{array}$} & \multicolumn{2}{|c|}{$\begin{array}{l}\text { Negative } \\
(\mathrm{N}=115)\end{array}$} & & \\
\hline & No & $\%$ & No & $\%$ & & \\
\hline \multicolumn{7}{|l|}{ Age group: } \\
\hline$\leq 18$ year & 9 & 20.5 & 35 & 79.5 & \multirow[t]{2}{*}{0.0002} & \multirow{3}{*}{$\begin{array}{l}4.18 \\
(1.89-9.24)\end{array}$} \\
\hline$>18$ years & 86 & 51.8 & 80 & 48.2 & & \\
\hline Mean \pm SD & \multicolumn{2}{|c|}{$43.7 \pm 19.0$} & \multicolumn{2}{|c|}{$35.4 \pm 24.6$} & $0.029 *$ & \\
\hline \multicolumn{7}{|l|}{ Gender: } \\
\hline Male & 57 & 47.9 & 62 & 52.1 & \multirow[t]{2}{*}{0.376} & \multirow{2}{*}{$\begin{array}{l}0.78 \\
(0.45-1.35)\end{array}$} \\
\hline Female & 38 & 41.8 & 53 & 52.2 & & \\
\hline \multicolumn{7}{|l|}{$\begin{array}{l}\text { Education }(>18 y r . \\
N=166) \text { : }\end{array}$} \\
\hline Lower & 15 & 53.6 & 13 & 46.4 & \multirow[t]{2}{*}{0.838} & \multirow{2}{*}{$\begin{array}{l}0.918 \\
(0.41-2.1)\end{array}$} \\
\hline Higher & 71 & 51.4 & 67 & 48.6 & & \\
\hline \multicolumn{7}{|l|}{$\begin{array}{l}\text { Occupation }(>18 y r ., \\
N=166) \text { : }\end{array}$} \\
\hline Non worker & 43 & 48.3 & 46 & 51.7 & \multirow[t]{2}{*}{0.333} & \multirow{2}{*}{$\begin{array}{l}1.353 \\
(0.73-2.49)\end{array}$} \\
\hline Worker & 43 & 55.8 & 34 & 44.2 & & \\
\hline \multicolumn{7}{|l|}{ Residence: } \\
\hline Urban & 55 & 41.7 & 77 & 55.3 & \multirow{2}{*}{0.176} & \multirow{2}{*}{$\begin{array}{l}1.47 \\
(0.84-2.59)\end{array}$} \\
\hline Rural & 40 & 51.3 & 38 & 48.7 & & \\
\hline \multicolumn{7}{|l|}{$\begin{array}{l}\text { Marital status } \\
(>18 y r ., N=166) \text { : }\end{array}$} \\
\hline Single & 8 & 66.7 & 4 & 33.3 & \multirow[t]{2}{*}{0.285} & \multirow{2}{*}{$\begin{array}{l}0.513 \\
(0.15-1.78)\end{array}$} \\
\hline Married & 78 & 50.6 & 76 & 49.4 & & \\
\hline \multicolumn{7}{|l|}{$\begin{array}{l}\text { Special habits } \\
\text { smoking: }\end{array}$} \\
\hline Smoker & 41 & 55.4 & 33 & 46.6 & \multirow[t]{2}{*}{0.03} & \multirow{2}{*}{$\begin{array}{l}1.89 \\
(1.02-3.49)\end{array}$} \\
\hline Non smoker & 54 & 39.7 & 82 & 60.3 & & \\
\hline
\end{tabular}

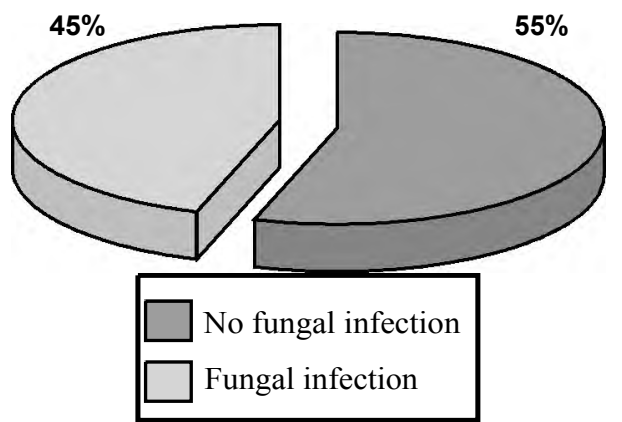

Fig. (1): Incidance rate for fungal infection. 
Table (2): Risk factors associated with fungal infection among patients undergoing hepatobiliary surgeries.

\begin{tabular}{|c|c|c|c|c|c|c|}
\hline \multirow{3}{*}{$\begin{array}{l}\text { Risk factors and } \\
\text { past history } \\
\text { of diseases }\end{array}$} & \multicolumn{4}{|c|}{ Fungal infection } & \multirow{3}{*}{$\begin{array}{c}\text { Chi } \\
\begin{array}{c}\text { square } \\
\text { test } \\
p \text {-value }\end{array}\end{array}$} & \multirow{3}{*}{$\begin{array}{c}\mathrm{OR} \\
(95 \% \mathrm{CI})\end{array}$} \\
\hline & \multicolumn{2}{|c|}{$\begin{array}{l}\text { Positive } \\
(\mathrm{N}=95)\end{array}$} & \multicolumn{2}{|c|}{$\begin{array}{c}\text { Negative } \\
(\mathrm{N}=115)\end{array}$} & & \\
\hline & No & $\%$ & No & $\%$ & & \\
\hline \multicolumn{7}{|l|}{$\begin{array}{l}\text { Long-term use of } \\
\text { antibiotic: }\end{array}$} \\
\hline Yes & 85 & 53.1 & 75 & 46.9 & \multirow[t]{2}{*}{0.00004} & \multirow{2}{*}{$\begin{array}{l}4.53 \\
(2.12-9.69)\end{array}$} \\
\hline No & 10 & 20.0 & 40 & 80.0 & & \\
\hline \multicolumn{7}{|l|}{ Chemotherapy: } \\
\hline Yes & 4 & 66.7 & 2 & 33.3 & \multirow[t]{2}{*}{0.41} & 2.48 \\
\hline No & 91 & 44.6 & 113 & 55.4 & & $(0.38-20.0)$ \\
\hline \multicolumn{7}{|l|}{ Steroids: } \\
\hline Yes & 13 & 72.2 & 5 & 27.8 & \multirow[t]{2}{*}{0.016} & 3.49 \\
\hline No & 82 & 42.7 & 110 & 57.3 & & $(1.2-10.17)$ \\
\hline \multirow{2}{*}{\multicolumn{7}{|c|}{$\begin{array}{l}\text { Immunosuppressive } \\
\text { drugs: }\end{array}$}} \\
\hline & & & & & & \\
\hline Yes & 6 & 85.7 & 1 & 14.3 & \multirow[t]{2}{*}{0.04} & 7.69 \\
\hline No & 89 & 43.8 & 114 & 56.2 & & $(0.89-172.49)$ \\
\hline \multicolumn{7}{|l|}{$H B V$ and/or $H C V$ : } \\
\hline Positive & 84 & 50.9 & 81 & 49.1 & \multirow[t]{2}{*}{0.002} & 3.21 \\
\hline Negative & 11 & 24.4 & 34 & 75.6 & & $(1.52-6.75)$ \\
\hline \multicolumn{7}{|l|}{ Diabetes: } \\
\hline Yes & 30 & 66.7 & 15 & 33.3 & \multirow[t]{2}{*}{0.001} & 3.08 \\
\hline No & 65 & 39.4 & 100 & 60.6 & & $(1.53-6.16)$ \\
\hline \multicolumn{7}{|l|}{ History of cancer: } \\
\hline Yes & 29 & 78.4 & 8 & 21.6 & \multirow{2}{*}{0.00008} & 5.88 \\
\hline No & 66 & 38.2 & 107 & 61.8 & & $(2.54-13.62)$ \\
\hline \multicolumn{7}{|l|}{ CMV infection: } \\
\hline Yes & 5 & 83.3 & 1 & 16.7 & \multirow[t]{2}{*}{0.093} & 6.33 \\
\hline No & 90 & 44.1 & 114 & 55.9 & & $(0.7-145.85)$ \\
\hline \multicolumn{7}{|l|}{ Anemia: } \\
\hline Yes & 73 & 49.7 & 74 & 50.3 & \multirow[t]{2}{*}{0.049} & 1.84 \\
\hline No & 22 & 34.9 & 41 & 65.1 & & $(1.00-3.39)$ \\
\hline \multicolumn{7}{|l|}{ Organ } \\
\hline \multicolumn{7}{|l|}{ transplantation: } \\
\hline Yes & 11 & 84.6 & 2 & 15.4 & 0.003 & 7.4 \\
\hline No & 84 & 42.6 & 113 & 57.4 & & $(1.60-34.27)$ \\
\hline Past operations: & & & & & & \\
\hline Yes & 78 & 59.1 & 54 & 40.9 & 0.0002 & 5.18 \\
\hline No & 17 & 21.8 & 61 & 78.2 & & $(2.73-9.82)$ \\
\hline
\end{tabular}

OR: Odds Ratio.

CI: Confidence Interval.

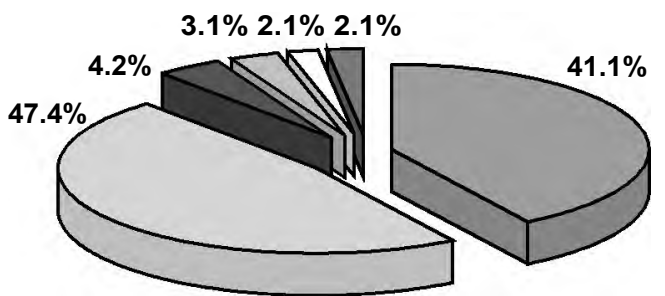
C. Albican
Mixed tropical \& C. albican
C. glaberata Mixed C. albican \& C. non albican
C. Tropiclis Asprigillus nigra

Fig. (2): Types of fungal infections.
Table (3): Invasive techniques risk factor in relation to fungal infection among patients undergoing hepatobiliary surgeries.

\begin{tabular}{|c|c|c|c|c|c|c|}
\hline \multirow{3}{*}{$\begin{array}{l}\text { Invasive } \\
\text { techniques }\end{array}$} & \multicolumn{4}{|c|}{ Fungal infection } & \multirow{3}{*}{$\begin{array}{c}\text { Chi } \\
\text { square } \\
\text { test } \\
p \text {-value }\end{array}$} & \multirow{3}{*}{$\begin{array}{c}\text { OR } \\
(95 \% \mathrm{CI})\end{array}$} \\
\hline & \multicolumn{2}{|c|}{$\begin{array}{l}\text { Positive } \\
(\mathrm{N}=95)\end{array}$} & \multicolumn{2}{|c|}{$\begin{array}{l}\text { Negative } \\
(\mathrm{N}=115)\end{array}$} & & \\
\hline & No & $\%$ & No & $\%$ & & \\
\hline \multicolumn{7}{|c|}{ Blood transfusion: } \\
\hline Yes & 55 & 66.3 & 28 & 33.7 & 0.0000007 & 4.27 \\
\hline No & 40 & 31.5 & 87 & 68.5 & & $(2.37-7.70)$ \\
\hline \multicolumn{7}{|l|}{$C V C:$} \\
\hline Yes & 80 & 65 & 43 & 35 & 0.0000001 & 8.93 \\
\hline No & 15 & 17.2 & 72 & 82.8 & & $(4.58-17.43)$ \\
\hline \multicolumn{7}{|l|}{ Intravenous } \\
\hline \multicolumn{7}{|l|}{ catheter: } \\
\hline Yes & 94 & 45 & 115 & 55 & 0.452 & 0.00 \\
\hline No & 1 & 100 & 0 & 0.0 & & $(0.00-14.39)$ \\
\hline \multicolumn{7}{|c|}{ Abdominal drain: } \\
\hline Yes & 89 & 48.9 & 93 & 51.1 & 0.007 & 3.51 \\
\hline No & 6 & 21.4 & 22 & 78.6 & & $(1.36-9.06)$ \\
\hline \multicolumn{7}{|c|}{ Urinary catheter: } \\
\hline Yes & 71 & 66.4 & 36 & 33.6 & 0.0000001 & 6.49 \\
\hline No & 24 & 23.3 & 79 & 76.7 & & $(3.54-11.92)$ \\
\hline \multicolumn{7}{|l|}{ Parenteral } \\
\hline Yes & 32 & 65.3 & 17 & 34.7 & 0.001 & 2.93 \\
\hline No & 63 & 39.1 & 98 & 60.9 & & $(1.50-5.71)$ \\
\hline \multicolumn{7}{|c|}{$\begin{array}{l}\text { Hospitalization } \\
I C U>48 h:\end{array}$} \\
\hline Yes & 83 & 61.5 & 52 & 38.5 & 0.0000001 & 8.38 \\
\hline No & 12 & 16.0 & 63 & 84.0 & & $(4.12-17.01)$ \\
\hline
\end{tabular}

Table (4): Logistic regression for the most relevant factors associated for developing fungal infection among patients undergoing hepatobiliary surgeries.

\begin{tabular}{|c|c|c|c|c|c|}
\hline \multirow{2}{*}{$\begin{array}{l}\text { Logistic } \\
\text { regression }\end{array}$} & $\beta$ & S.E. & $\begin{array}{l}\text { Wald } \\
\text { test }\end{array}$ & $\begin{array}{c}p- \\
\text { value }\end{array}$ & $\begin{array}{c}\text { OR } \\
\text { (CI 95\%) }\end{array}$ \\
\hline & \multicolumn{5}{|c|}{3 constant $=7.17$} \\
\hline - Age category. & 1.594 & 0.485 & 10.80 & 0.001 & $\begin{array}{l}4.924 \\
(1.903-12.739)\end{array}$ \\
\hline - Antibiotic use. & 0.913 & 0.485 & 3.55 & 0.05 & $\begin{array}{l}2.491 \\
(0.964-6.437)\end{array}$ \\
\hline $\begin{array}{l}\cdot \text { HBV and/or } \\
\text { HCV infection. }\end{array}$ & 1.376 & 0.496 & 7.70 & 0.006 & $\begin{array}{l}3.958 \\
(1.498-10.457)\end{array}$ \\
\hline $\begin{array}{l}\text { - Central venous } \\
\text { catheter using. }\end{array}$ & 0.949 & 0.469 & 4.09 & 0.043 & $\begin{array}{l}2.583 \\
(1.030-6.476)\end{array}$ \\
\hline - Urinary catheter. & 0.831 & 0.433 & 3.68 & 0.05 & $\begin{array}{l}2.295 \\
(0.983-5.358)\end{array}$ \\
\hline $\begin{array}{l}\text { - Hospitalization } \\
\text { ICU > } 48 \mathrm{hr} \text {. }\end{array}$ & 1.770 & 0.456 & 15.09 & 0.000008 & $\begin{array}{l}5.874 \\
(2.405-14.347)\end{array}$ \\
\hline
\end{tabular}

\section{Discussion}

In the present study the incidence rate of fungal infection among patients undergone major hepatobiliary surgeries in national liver institute is $(45.2 \%)$ which is consistent with study of Magalhães et al., [5] at three different hospitals were (39\%), $(29.6 \%)$ and C (31.4\%) [5]. The Singh et al., [6] study who found fungal infection rate was only $11 \%$ out of 
the 130 liver transplanted patients [6]. In this study $45.2 \%$ of the patients were positive culture for fungal infection, nearly $98 \%$ of positive culture were of candida species only this was in agreement with Magalhães et al., [5] study which showed that $89.8 \%$ of all the isolates (97 isolates) belonged to the Candida genus. In this study age, antibiotic use, liver disease, central venous catheter, urinary catheter, and ICU hospitalization more than 48 hours were the independent predictors of fungal infection, however in the study of Wang et al., [7] the distribution of Candida species did not differ between elderly (above 60) and younger patients (below 60) which is against the result of the current study. In the current study long term use of antibiotics by hepatobiliary surgery patients' was two times risky to acquire fungal infection than others. This was consistent with Cornely et al., [8] study which stated that the risk of fungal infection also increased in patients who received a high number of antibiotics ( $p$-value 0.019) or long term using [9] however it was in contrast to study of Blumberg et al., [9] study where there was no increased risk of developing fungal infection associated with the individual use of some antibiotic agents. In the current study positive HBV and/or HCV infection was four times risky to acquire fungal infection than others, this result was compatible with the study of Singh et al., [6] which sated that histopathological document of recurrent hepatitis $C$ virus hepatitis was a significant factor associated with late fungal infections in liver transplant patients $(p=0.006)$. In the current study central venous catheter usage was three times risky to acquire fungal infection than others where this result was similar to result of Blumberg et al., [9] study which stated that patients who underwent a surgical procedure or had a CVC were 11 times more likely to develop candidemia. In the current study urinary catheter usage was two times risky to acquire fungal infection than others which was in consistent with Tambyah and Maki [10] concluded that $14.9 \%$ of newly catheterized patients developed catheter associated urinary tract infections. The current study stated that hospitalization Intensive Care Unit more than 48 hour was six times risky to acquire fungal infection than others which was compatible with Blumberg et al., [9] study in which $76.0 \%$ of CBSI cases occurred within the first 3 weeks of admission to an surgical Intensive Care Unit. Also Wade et al., [11] study noticed that patients with early fungal infections had prolonged ICU admissions more than 48 hours $p$-value was 0.002 .

Conclusions and Recommendations: Based on the findings of the present study, we can conclude that, high incidence of fungal infection after major hepatobiliary surgeries may reach $45 \%$ due to type of patient doing such surgeries. The great prevalence of fungal colonization inside ICU which is easily transmissible emphasizes very strongly on the importance of infection-control guidelines. Aggressive antibiotics shouldn't be used outside ICU and only antibiotics according to culture and sensitivity should be used to lessen down emergence of resistant strains and fungal flourishment. Using of antifungal prophylaxis only to high risk patients as a routine use of antifungal prophylaxis may cause resistance of fungal pathogens and selection of more hostile and less susceptible fungal species. A great attention must be paid in Egyptian hospitals for patients who undergoing major hepatobiliary surgeries in order to prevent and treat the fungal infections. Continued epidemiologic and laboratory investigations is needed to better characterize the ever-increasing array of endemic and opportunistic fungal pathogens, allowing for improved diagnostic, therapeutic and preventive strategies in the future.

\section{References}

1- United States Centers for Disease Control. CDC and Fungal Diseases, http://www.cdc.gov/ncezid/dfwed/pdfs/ fungal-factsheet-508c.pdf, 2011.

2- SINGHI S. and DEEP A.: "Invasive candidiasis in pediatric intensive care units." Indian J. Pediatr., 76 (10): 1033 44, 2009.

3- OSTROSKY-ZEICHNER L., REX J.H., PAPPAS P.G., et al.: "Antifungal susceptibility survey of 2,000 bloodstream Candida isolates in the United States." Antimicrob Agents Chemother., 47 (10): 3149-54, 2003.

4- PFALLER M.A., PAPPAS P.G. and WINGARD J.R.: "Invasive Fungal Pathogens: Current Epidemiological Trends.", Clin. Infect. Dis., 43 (Supplement 1): S3-S14, 2006.

5- MAGALHÃES, YANKEE C., BOMFIM, MARIA ROSA Q., MELÔNIO, LUCIANE C., et al.: "Clinical significance of the isolation of Candida species from hospitalized patients", Brazilian J. Microbiol., 46 (1): 117-23, 2015.

6- SINGH N., GAYOWSKI T., WAGENER M.M., DOYLE H. and MARINO I.R.: "Invasive fungal infections in liver transplant recipients receiving tacrolimus as the primary immunosuppressive agent." Clin. Infect. Dis., 24 (2): 17984, 1997.

7- WANG H., WANG, HAO, LIU, NAIZHENG, YIN, MEI, HAN, et al.: "The epidemiology, antifungal use and risk factors of death in elderly patients with candidemia: A multicentre retrospective study", 1-9, 2014.

8- BLUMBERG H.M., JARVIS W.R., SOUCIE J.M., EDWARDS, et al.: "Risk factors for candidal bloodstream infections in surgical intensive care unit patients: The 
NEMIS prospective multicenter study.", The National Epidemiology of Mycosis Survey. Clin. Infect. Dis., 33 (2): 177-86, 2001.

9- CORNELY, OLIVER A., BÖHME, ANGELIKA, REICHERT, DIETMAR, et al.: "Risk factors for breakthrough invasive fungal infection during secondary prophylaxis.", J. Antimicrob. Chemother., 61 (4): 939-46, 2008.
10- TAMBYAH P.A. and MAKI D.G.: "Catheter-Associated Urinary Tract. Infection Is Rarely Symptomatic", 2013.

11- WADE J.J., ROLANDO N., HAYLLAR K., PHILPOTTHOWARD J., CASEWELL M.W. and WILLIAMS R. "Bacterial and fungal infections after liver transplantation: An analysis of 284 patients." Hepatology, 21 (5): 132836, 1995.

\section{العدوى الفطرية فى العمليات الجراحية الكبدية العيدية بعد العمليات الجراتيات العماتية الوبائينات (عوامل الإنتشار والمخاطر)}

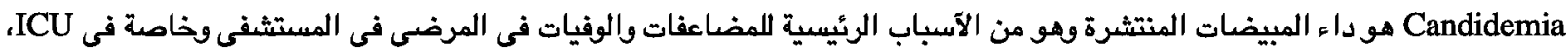

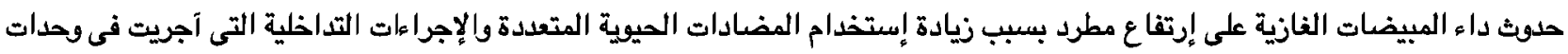

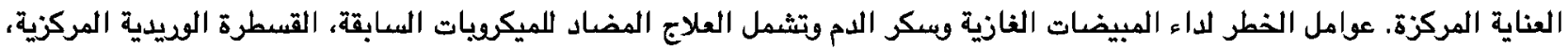

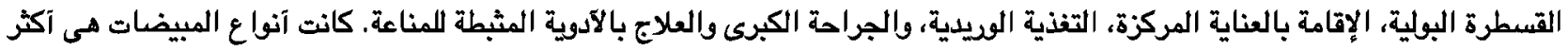

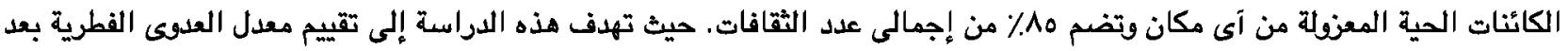

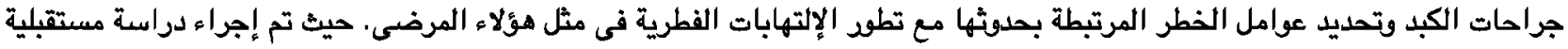

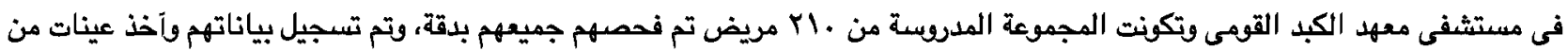

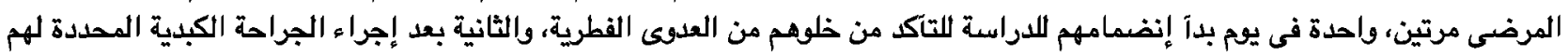

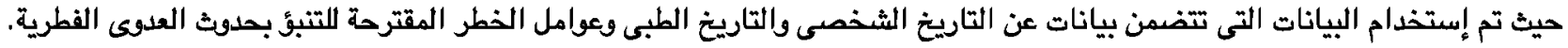

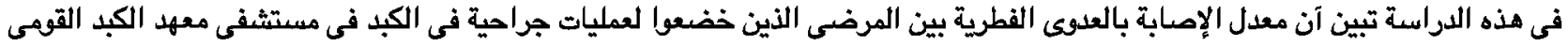

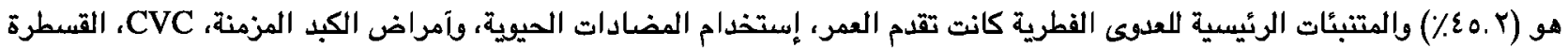

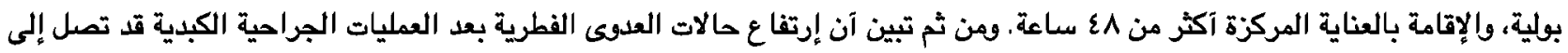

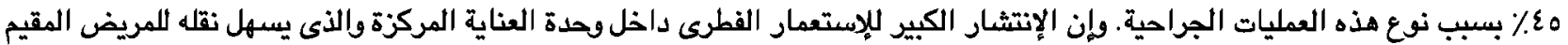

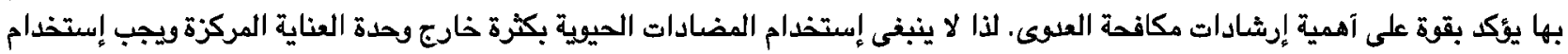

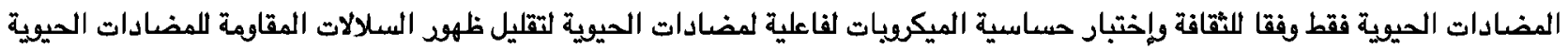

